\title{
Macular hole with central retinal detachment in high myopia with posterior staphyloma
}

\author{
ABDEL-LATIF SIAM \\ Ophthalmology Department, Ein Shams University, Cairo, Egypt
}

Advanced chorioretinal atrophy and vascular sclerosis are common findings in hïgh myopia, particularly in association with a posterior staphyloma. Cystic macular degenesation followed by hole formation is therefore more common in eyes with high myopia than in other eyes, and probably most frequent in cases of high myopia with posterior staptylomata, a fact which does not appear to have been pointed out before. Macular holes-in posterior staphylomata are very likely to be missed, and it may be impossible to diagnăse them for various reasons :

(I) Difficult ophthalmoscopy with deep posterior staphylomata.

(2) Lack of contrast at the bottom of deep staphylomata with extreme chorioretional atrophy.

(3) The general difficulty of diagnosing all macular holes.

This is borne out by the clinical "disappearance" of the hole after drainage of the s感retinal fluid in cases of posterior staphylomata with macular hole and central reti $\vec{\pi}$ al detachment.

In my experience all cases of macular holes in posterior staphylomata have been aş్sciated with central retinal detachment. The hole may have been present for a long time before the central retinal detachment developed, and the patients all gave a history tha the affected eye had gradually become weak, with more recent deterioration and obscuaㅡ. tion of vision.

The clinical diagnosis is relatively easy after the detachment as the hole stands outin contrast to the surrounding grey retina, and parallax with the slit lamp and even wsth the binocular indirect ophthalmoscope becomes valuable in diagnosis. Macular hotes do not necessarily lead to retinal detachment, but all the macular holes in cases of cental retinal detachment occurred in patients with deep posterior staphylomata. I have wot seen a case of macular hole and central retinal detachment in eyes without staphylomeda except in association with peripheral retinal breaks and peripheral detachment, in whid h the diagnosis of a macular hole is very doubtful even after slit-lamp fundoscopy (Rosengren, I966). Rosengren stated that most cases were excessively myopic and that the elevan affected only the posterior parts of the fundus while the periphery remained intact. Ф़e did not mention posterior staphyloma though it appears that the condition was presentin all his cases.

The diagnosis of central retinal detachment due to a macular hole with a deep posterior staphyloma is very difficult with direct ophthalmoscopy; the thinned central ret渵a stretches across the cavity of the deep posterior staphyloma which is filled with subretifal 
fluid, and the detachment hardly extends beyond the limits of the cavity. The retinal folds are few and fine, the retinal vessels are not very tortuous, and the macular hole is sometimes very difficult to find in the thinned central retina with a very pale and atrophic central choroid in the background. With the binocular indirect ophthalmoscope, however, the diagnosis becomes easy. The image is clear and well illuminated, the field is much wider, the edges of the posterior staphyloma appear sharply defined, and the wide separation between the bottom of the posterior staphyloma and the retina stretched across it is seen stereoscopically. Examination of the other eye usually shows a posterior staphyloma and there is a marked contrast between the ophthalmoscopic pictures on the two sides.

\section{Treatment}

The best method consists in a volume-reducing operation and the complete removal of the subretinal fluid by a diathermy puncture followed by photocoagulation of the macular hole. Four cases have been successfully operated on in this way. Volume reduction was achieved by an Arruga suture in two cases and by scleral infolding in the other two. The subretinal fluid was completely removed, but the amount of fluid was unexpectedly large and this has to be taken into consideration when planning a scleral infolding operation. The infolding should be long and broad enough to allow complete evacuation of the subretinal fluid and restore firm tension.

It was easy to reach the posterior staphyloma by passing a stitch under the inferior oblique muscle. The staphyloma presented as a localized, bluish, sac-like bulge at the posterior pole of the eye. An electrolysis puncture was used in the first two cases, but in both a small choroidal haemorrhage oozed centrally. In the other two cases diathermy puncture was used and no choroidal haemorrhage occurred.

Other methods Rosengren (1966) devised the silver ring and plomb method. The silver ring is attached concentrically with the limbus, and an arm projecting from the ring extends inwards over the eyeball with a silver ball $8 \mathrm{~mm}$. in diameter at its tip. If the ball is exactly placed in the foveal region and is made to press the posterior pole so that the macular hole is exactly at its apex, the subretinal fluid will vanish in a day or two, and light coagulation can be carried out. The silver ring and plomb are removed after 4 weeks.

Blach (1966) placed a small silastic plomb over the hole (removing fluid only if chorioretinal contact was not obtained) and then applied light coagulation to the hole. $\mathrm{He}$ pointed out that access to the macular region was not difficult if the lateral rectus muscle was removed.

\section{Summary}

(I) The particularly high incidence of macular holes in cases of high myopia with deep posterior staphyloma is pointed out.

(2) Four cases of central detachment due to macular hole were all excessively myopic with very deep posterior staphylomata.

(3) The reasons for this combination are discussed and methods of treatment described.

\section{References}

BLACH, R. K. (I966) Discussion of Rosengren (I966)

ROSENGREN, B. (ig66) Trans. ophthal. Soc. U.K., 86, 49 